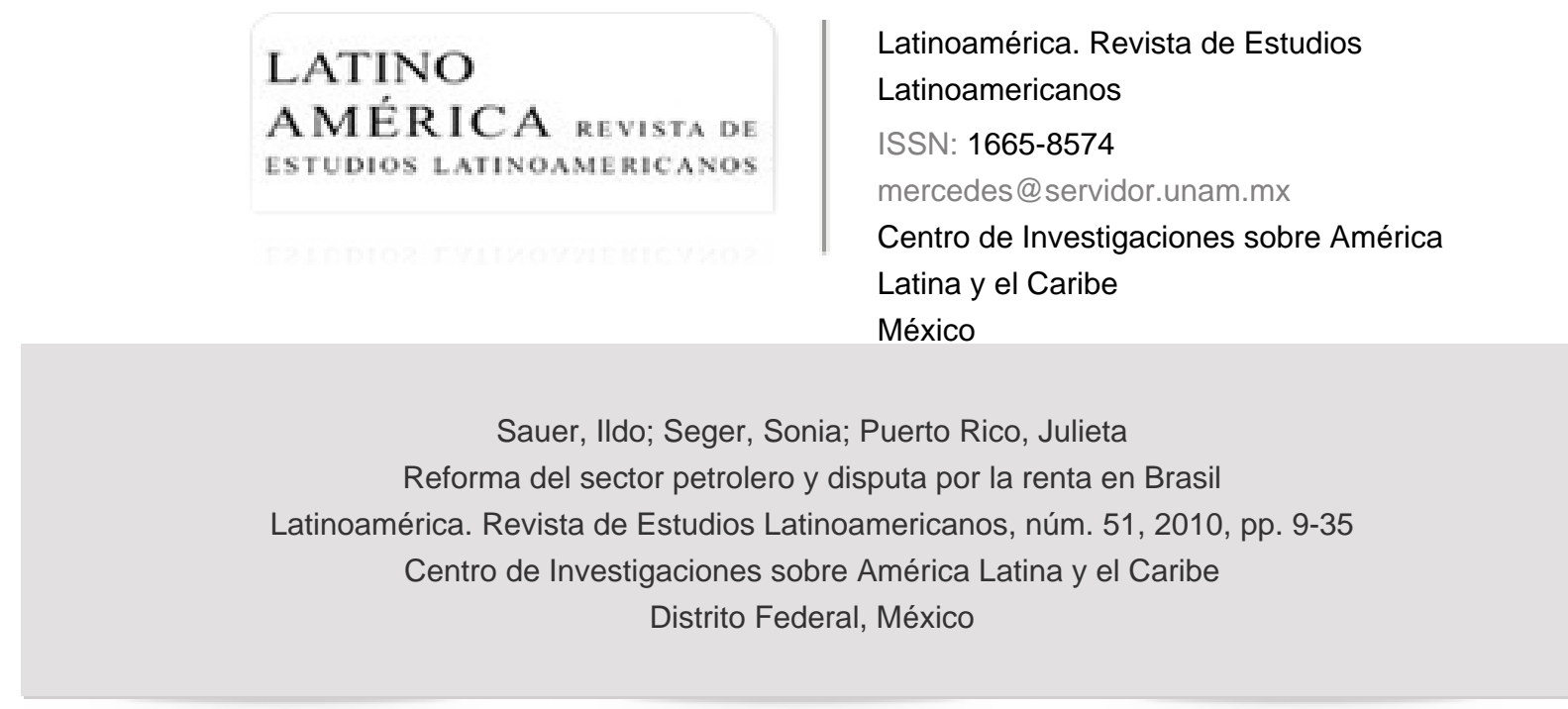

Disponible en: http://www.redalyc.org/articulo.oa?id=64015153002

- Cómo citar el artículo

- Número completo

- Más información del artículo

- Página de la revista en redalyc.org

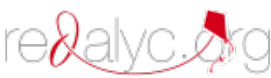

Sistema de Información Científica

Red de Revistas Científicas de América Latina, el Caribe, España y Portugal Proyecto académico sin fines de lucro, desarrollado bajo la iniciativa de acceso abierto 


\section{Reforma del sector petrolero y disputa por la renta en Brasil}

Ildo Sauer*, Sonia Seger** y Julieta Puerto Rico***

Resumen: El artí́culo analiza el proceso de reforma liberal del sector petrolero brasileño y la disputa por la renta generada. Ese mismo proceso de reestructuración de la producción y uso de mecanismos del mercado petrolero facilitó la apropiación de la renta de este sector a través de la ruptura del monopolio de la empresa estatal, aplicándose el modelo de concesiones, situación que ha incidido en el intento de reformar la legislación del sector petrolero a partir de proyectos de Ley enviados al Congreso de Brasil, en los que se pretende la creación de una nueva empresa, la Petrosal, de un Fondo Social y la aplicación de un régimen de producción compartida que permita la participación de las empresas extranjeras y de empresas privadas "nacionales".

PalabRas clave: Sector petrolero, Reforma sectorial, Renta petrolera.

ABSTRACT: The article analyzes the process of liberal reform of the petroleum Brazilian sector and the dispute for the generated revenue. The same process of restructuring of the production and use of mechanisms of the petroleum market, it facilitated the appropriation of the revenue of this sector across the break of the monopoly of the state company, being applied the model of concessions, situation who has affected in the attempt of reforming the legislation of the petroleum sector from a series of projects of law sent to the Congress of Brazil, in that there is claimed the creation of a new company, the Petrosal, the creation of a Social Fund and the application of a regime of shared production that allow the participation of the foreign companies and of "national" private companies.

Key Words: Oil Industry, Sectoral Reform, Oil Income.

Instituto de Electrotécnica y Energía, Universidad de Saõ Paulo (illsauer@iee.usp.br).

** Instituto de Electrotécnica y Energía, Universidad de Saõ Paulo (seger@ups.br).

*** Instituto de Electrotécnica y Energía, Universidad de Saõ Paulo (julietapuerto@gmail.com). 
ENERGíA, SOCIEDAD Y EL PAPEL DEL PETRÓLEO

$\mathrm{L}$

a historia de la humanidad guarda profundos vínculos con el proceso de apropiación social de la energía. Nuestra especie, el Homo sapiens, durante más de 200 mil años se dedicó a cazar y recoger todos aquellos recursos que le ofreció la naturaleza para luego transformarlos en fuentes de energía a partir de procesos conocidos como la fotosíntesis, auxiliada entonces por los ciclos solares e hidrológicos de los que da cuenta la ciencia.

De este modo surgieron también las sociedades agrarias, las cuales se encargaron de poblar los distintos continentes, y con ello estructurar nuevas formas de organización social regidas por el trabajo del hombre y su relación directa con la naturaleza. En ese mismo proceso se darán a conocer siglos más tarde revoluciones del pensamiento, como las que emprendieron los ingleses, cuya base energética consistió en el aprovechamiento del carbón para el calentamiento del agua, y la producción de vapor para accionar émbolos y mover máquinas, telares, trenes y navíos.

Técnicas tan trascendentales como éstas se convertirían en la base fundamental para el desarrollo de la producción capitalista de los últimos tiempos, solventada en un nuevo régimen de trabajo a través de lo que se conoce como "mano de obra asalariada". De este modo, el sector campesino y rural optó por trasladarse del campo a la ciudad para convertirse en asalariados de los medios de producción de aquellas fábricas citadinas, que requerían y demandaban un mayor número de trabajadores para hacer más eficiente la producción con el manejo de las máquinas.

Derivada de esta primera etapa aparecerá la segunda revolución del sistema capitalista a finales del siglo xIx, precisamente con la aparición de las telecomunicaciones, el generador eléctrico, el motor de combustión interna a base de gasolina y diesel, que de paso sirvió para sustituir los medios de transporte tradicionales como habían sido el caballo y los carruajes, dando lugar al inicio formal de la industria del automóvil. Este hecho de comienzos del siglo xx, se convertiría en el puntal de una nueva fase del capitalismo y de transformación social a partir de la conformación de nuevos emporios comerciales asociados al sistema financiero. Se dio entonces una extraordinaria intensificación de la pro- 
ducción de bienes y mercancías, acompañados de un proceso de circulación y de consumo a gran escala de recursos vitales para la naciente industrialización, todo ello fincado en la extracción del petróleo y de sus derivados.

Fue de esta manera como el petróleo se convertiría desde entonces en la principal fuente de energía aplicada al modo de vida urbano-industrial del siglo xx, la cual persiste hasta los tiempos actuales. De ahí su enorme trascendencia, pero también su relevancia en los procesos políticos mundiales y en los que cada país lleva a cabo. En ese sentido, cabe destacar los siguientes datos, cuando la industria petrolera comenzó, la energía líquida disponible estaba en razón de 1 para 100, es decir, se gastaba un barril de petróleo para obtener 100 barriles. Hoy en día esta proporción está dada en razón de 1 para 30, lo cual significa que se gasta en capital y trabajo humano el equivalente a un barril de petróleo para producir apenas 30 barriles. ${ }^{1}$

El problema de ese costo cada vez mayor debe verse comparativamente. La fuente alternativa al petróleo que hoy resulta más competitiva es el etanol brasileño, el cual tiene una relación de 1 para 8 . Mientras que el biodiesel producido a partir de aceites vegetales es de 1 para 1 . La conversión directa del sol en electricidad, que es energía fotovoltaica, tiene una relación semejante. Hoy el petróleo se produce a un costo de 1 a 10 dólares el barril equivalente. Su valor en el mercado osciló en los últimos años entre 60 y 150 dólares el barril, lo cual significa un excedente enorme de más de 50 dólares por barril. Surge así la renta diferencial, disputada en el campo económico, político e ideológico de los estados y las grandes empresas. Al respecto cabe señalar que el sistema económico mundial consume cerca de 30 mil millones de barriles al año, ${ }^{2}$ permitiendo la apropiación de un excedente económico del orden de dos billones de dólares anuales.

${ }^{1}$ Estimación de los autores con base en el balance de energía neta y costos de producción por barril de petróleo.

2 British Petroleum (BP), Statistical Review of World Energy 2010. En http://www.bp.com/ liveassets/bp_internet/globalbp/globalbp_uk_english/reports_and_publications/statistical_ene rgy_review_2008/STAGING/local_assets/2010_downloads/Statistical_Review_of_World_Ene rgy_2010.xls (fecha de consulta: junio de 2010). 
Sin embargo, no es correcto vincular el problema del alto costo del petróleo al hecho de ser una fuente natural, esta situación radica en la sociedad y en su organización para la producción. La demanda total de petróleo no es determinada a partir de un país, sino a partir de la forma como la organización mundial de la producción establece sus precios y sus niveles de circulación, junto con el de las personas en todo el mundo. El petróleo continúa ejerciendo un papel esencial para el modelo de producción capitalista. Esto hablando del mundo real de las sociedades urbanizadas de hoy, con enormes industrias automovilísticas en los países ricos y en crecimiento de la talla de países como China. Lo cual nos lleva a formular la pregunta ia qué se le atribuye esa característica especial del petróleo?

En la actualidad, el recurso energético de mayor disponibilidad en stocks resulta ser el carbón, lo mismo que el uranio. Ahora, en términos de la cantidad de energía que llega a la tierra proveniente del sol y que vuelve al espacio después de algunas transformaciones es inmensa. Cada una de las tres formas en que la energía solar asume su acción sobre la tierra - la energía hidráulica, la eólica, y la de la fotosíntesis - requiere de un mayor valor stock de petróleo acumulado. Sin embargo, en función del papel que el petróleo obtuvo en el sistema urbano industrial, que apareció a partir de la Segunda Revolución Industrial, ningún recurso energético contribuye más que éste para hacer girar la rueda de consumo. A su vez, el consumo mueve la rueda de producción que hace funcionar cada vez más rápido la máquina de generación de excedente.

Por ello otra pregunta a resolver deriva del siguiente planteamiento: ise pueden imaginar cambios en ese modelo urbano-industrial y la transición basados en otro modelo de menor uso de energía? Para que otras formas de energía desempeñen ese mismo papel es necesario mejorar las condiciones técnicas de su apropiación, para que éstas usen menos capital y menos trabajo vivo. En ese sentido, los economistas ecológicos hablan de la necesidad de cambiar ese paradigma, consideran que es necesario, posible, pero llevará tiempo, debido a que no existe en este momento una fuerza política global capaz de asegurar el establecimiento de dicho paradigma. 
Está en curso también un proceso de transición energética, provocado por la discusión del cambio climático y por la perspectiva de agotamiento de las reservas de petróleo, ya que el ritmo de descubrimientos de nuevos yacimientos no es suficiente comparado con el crecimiento del consumo. De todas formas, cuando se observa la estructura social de producción, la persistencia del modelo de desarrollo urbano industrial, surgido de las revoluciones industriales, definitivamente se encuentra ligado al extraordinario desempeño que tiene el petróleo; a pesar de que existen dos razones que justifican la necesidad de una transición energética hacia nuevas fuentes renovables. La primera es el propio agotamiento del petróleo, y la segunda es la necesidad de enfrentar el cambio climático.

En lo que concierne al primer problema, es un hecho que no puede evitarse, porque los recursos de petróleo convencionales están agotándose en razón de la tasa actual de consumo, que está próxima a 85 millones de barriles de petróleo por día. ${ }^{3}$ Esto significa que los dos billones de barriles remanentes de recursos conocidos de petróleo convencionales se estarán agotando de cualquier manera en las siguientes tres o cuatro décadas, dado que el consumo y la producción están aumentando, a pesar de la problemática del cambio climático, con la matriz carbonizada de la economía mundial, y las tentativas de buscar nuevas fuentes de energía que permitan sustituir el petróleo en función de su agotamiento tomando en cuenta la reducción de emisiones de gases de efecto invernadero.

La solución simultánea de los dos problemas exige también inversión en ciencia y tecnología, para atenuar los impactos que esta sustitución va a tener en la estructura de producción y de gasto. Por ejemplo, será necesario implementar un cambio en los patrones de consumo de las actuales sociedades, sugiriendo un menor uso del automóvil como medio de transporte individual; con lo cual ese cambio de conducta social llevará de la mano otras iniciativas no sólo en materia productiva sino medioambiental, la cuestión de la polución, entre otros.

Sobre el caso particular de estudio que es Brasil, cabe señalar que su estructura productiva estará determinada para sus 190 millones de habitantes, la cual no dista mucho de enfrentarse, como el resto de la humanidad, al gran desafío de otras sociedades del mundo - de cómo producir más y distribuir mejor la pro-

3 Loc. cit. 
ducción para atender las necesidades de gran parte de la población-, haciendo uso de fuentes de energía con menores impactos a la atmósfera, que no disminuya la productividad del sistema económico reduciéndolo simplemente a la acumulación de excedentes.

La solución de este dilema pasa necesariamente por la alteración del patrón de consumo, por el aumento y mejora en la distribución de la producción, lo que implica la necesidad de elevar la productividad del trabajo y del capital; el uso de fuentes de energía como el petróleo, que tendrán todavía un enorme valor mientras persistan las características básicas del actual modelo de desarrollo urbano industrial, en medio de una persistente transición hacia el uso de nuevas fuentes energéticas, en las que el petróleo mantendrá su elevado valor por largo tiempo, tres o cuatro décadas como mínimo. Lo que da cuenta de que quien controle la apropiación de este recurso estratégico, en cualquier parte del mundo, tendrá en su haber un importante recurso de poder.

De esta manera cabe preguntarnos idónde se encuentra el remanente del petróleo? Y la respuesta indica que éste se ubica en tres fronteras estratégicas: una en Asia Central; otra en África, en países como Nigeria y Sudán, y ahora en el área conocida como Pré-Sal brasileño. Esto da una idea de lo que está en juego. La importancia política de la intervención estatal como forma de apropiarse de parte de la renta extra creada por el petróleo es relativamente reciente, si bien la intervención estatal en la economía es más antigua, se puede señalar al respecto que en el caso del petróleo, ésta surge en 1938, en México, con la creación de la empresa estatal Pemex. Años después con la creación de la Organización de Países Exportadores de Petróleo (OPEP) en 1960, cuando se comenzó a hablar del problema de la apropiación de la renta petrolera, tal como ocurrió con los choques y variaciones de precios del petróleo entre 1973 y 1978.

Será entonces la renta lo que está en disputa, no sólo en Brasil sino en todos los rincones del mundo. En el caso particular de Brasil, el Congreso Nacional, como será explicado en el apartado 4, tiene actualmente la responsabilidad extraordinaria de decidir a quienes se otorgarán las grandes rentas generadas a partir de los recursos del Pré-Sal, en una de las más grandes fronteras del petróleo en el ámbito mundial. 
PETROBRAS: URBANIZACIÓN, INDUSTRIALIZACIÓN, AUTOSUFICIENCIA Y DESCUBRIMIENTO DEL PRÉ-SAL

La percepción del papel de la apropiación social de la energía, especialmente del petróleo y de la industria eléctrica en los procesos de transformación social, inducidos por la industrialización y urbanización, se debió en buena medida a la lucha de los brasileños entre las décadas de 1940 y 1950, quienes incidieron en la formación del monopolio estatal del petróleo y en la creación de la empresa Petrobras, pero además en la conformación de la empresa eléctrica Eletrobras, otra como Telebras, el Banco Nacional de Desarrollo Económico (BNDE), la Compañía Siderúrgica Nacional (CSN), consideradas como instrumentos indispensables para la transformación de una sociedad agrario-mercantil con miras a un mayor y mejor progreso de sus sectores productivos.

En ese contexto de los años cuarenta y cincuenta fue justo cuando se logró percibir la importancia de tener el dominio de la energía para el proceso de modernización productiva, en medio de una campaña que se conoció como: "El petróleo es nuestro", y que daría la coyuntura idónea para la creación de la empresa Petrobras. En cuya primera fase, ésta tuvo a bien garantizar que todas las regiones del país tuvieran acceso a los derivados del petróleo, un factor esencial para la modernización y la mejora en las condiciones de vida de los brasileños. A su vez, fue creada con el objetivo de promover nuevos descubrimientos de petróleo para abastecer el mercado interno, ya que la producción nacional resultaba insuficiente para la época, sólo cubría 1.6\% del consumo total.

De tal forma que se tomó la decisión de ampliar el sector de refinación existente, a fin de reducir los costos de las importaciones de los derivados de petróleo. En ese sentido, Petrobras cumplió con dicho objetivo, lo que incidió para que la compañía intensificara la exploración y el trabajo en la formalización y especialización de su cuerpo técnico. En el esfuerzo de garantizar el abastecimiento, la empresa pasó a desarrollar actividades fuera de Brasil y descubrió, en esta fase, el mayor campo petrolífero de Irak, llamado Majnoon (el loco) dada su dimensión, campo que fuera nacionalizado. ${ }^{4}$

${ }_{4}^{4}$ Ricardo Pinho, Gigantes brasileiros: multinacionais emergentes e competição global, 2008 (Tesis de doctorado, Escuela de Administración de Empresas, Fundación Getulio Vargas, São Paulo). 
Con el primero y el segundo choque petrolero en 1973 y 1979, respectivamente, se generó una situación, según la cual la economía mundial entró en crisis. El paradigma keynesiano de intervención estatal también se vio afectado por la crisis, pues las tasas de acumulación de capital se redujeron drásticamente. Países como Brasil, que se habían embarcado en un proceso de desarrollo acelerado, soportado con financiamiento externo, se vio doblemente amenazado ante una factura petrolera extremadamente costosa, así como por la inflación internacional combinada con las altas tasas de interés, consecuencia de la crisis estadounidense de los años ochenta. Esas condiciones llevaron a Brasil a enfrentar un inédito escenario de crisis, dentro del cual Petrobras fue comisionada a una nueva misión, la que consistió en lograr la autosuficiencia petrolera al aminorar la dependencia energética, al realizar trabajos de exploración en tierra, pero también en su área marítima.

Fue entonces cuando en 1968 se iniciaron las primeras actividades de prospección offshore (costa afuera), en el recién descubierto campo de Guaricema, Sergipe. Más tarde, en 1974 se descubrió la cuenca Campos que es, hasta el momento, la mayor productora de hidrocarburos en Brasil. El área inicial fue Garoupa, seguida por los campos gigantes de Marlin, Alacora, Barracuda y Roncador. ${ }^{5}$ Precisamente fue en esta fase que se desarrolló la tecnología de exploración en aguas profundas y ultra profundas. Progresivamente, la exploración en láminas de agua de pocas decenas de metros pasó a medirse en centenas y, posteriormente, en 1 000, 2000 y hasta $3000 \mathrm{~m}$ de profundidad. De esta forma Brasil alcanzó los estándares de autosuficiencia hasta 2006, lo que de paso le permitió no sólo su autosuficiencia sino, además, la estabilidad macroeconómica necesaria para un país con las dimensiones poblacionales y territoriales de Brasil, en momentos en que el precio del petróleo superó los 100 dólares por barril de petróleo.

El acierto de la estrategia de Petrobras ha consistido en invertir grandes capitales en exploración y producción al interior de Brasil, pero también en el extranjero al procurarse una tendencia a la valorización definitiva del petróleo en

\footnotetext{
5 J. O. Neto y A. J. Costa, "A Petrobras e a exploração de petróleo offshore no Brasil: um approach evolucionário", en Revista Brasileña de Economía, vol. 61, núm. 1, Río de Janeiro, enero-marzo, 2007.
} 
el escenario del preagotamiento, a pesar de las restricciones impuestas en la actualidad ante los escenarios que plantea el cambio climático.

En ese mismo contexto, la explotación del gas puede verse como una posibilidad adicional de generar riqueza, ya que de cada 150 metros cúbicos de extracción de gas se permite la sustitución de un barril de petróleo, además de facilitar una descarbonización progresiva del mismo. Pero el esfuerzo en el segmento de biocombustibles y de otras fuentes renovables como la eólica y la fotovoltaica constituyen la base para crear, desde ahora, una alternativa del agotamiento final y una respuesta definitiva para la descarbonización de la matriz energética.

De este modo, la estrategia a considerar surge de un trabajo de años, cuyo gran patrimonio no es simplemente el hecho de descubrir el petróleo, sino también de potencializarlo, desarrollarlo, procesar gas natural y brindar soluciones hacia una inevitable transición de este tipo de energéticos, dentro de lo que se considera la era pospetróleo, que incluye por supuesto los biocombustibles y otras fuentes renovables conocidas en estos momentos. Por eso el valor adicional de Petrobras radica en la incorporación de 75000 personas que han encabezado un esfuerzo histórico del pueblo brasileño que creyó en esta empresa, al ofrecerle todo su apoyo en medio de la amenaza de privatizarse, en el pleno auge del neoliberalismo despuntado a partir de los años noventa.

La capacitación en el área de exploración, desarrollo, producción, gestión asociada a la interacción con grandes organizaciones mundiales de punta, permitieron a Petrobras probar un nuevo modelo geológico, desarrollado a lo largo de décadas, que preveía la posibilidad de la existencia de una segunda capa de petróleo, bajo la capa de sal, debajo de la primera; lo cual permitiría alcanzar la autosuficiencia deseada. El primer descubrimiento de petróleo de Pré-Sal se presentó en el bloque de Parati, en 2005. Se trató de un primer pozo petrolero con importantes resultados, sin embargo, fue el 1-RJS-628A de Tupi, cuya perforación del pozo pionero comenzó en septiembre de $2005,{ }^{6}$ el que constituyó la oportunidad para probar el nuevo modelo geológico que venía siendo construido desde hace muchos años, y que mostraba la posibilidad de encontrar mucho petróleo en un nivel más profundo, en Pré-Sal.

${ }^{6}$ Petrobras, Balanço social e ambiental 2008, Río de Janeiro, 2009. 
De este modo, la empresa se mantuvo en el entendido de continuar con las exploraciones profundas, fue entonces que a comienzos de julio se realizó el gran descubrimiento, si bien era apenas un pozo, en mayo de 2007 se logró perforar el pozo de extensión 3-RJS-646. Cuando se descubrió petróleo, se confirmó el enorme potencial del yacimiento, evaluado después entre 4 y 8000 millones de barriles de petróleo leve, equivalente a uno o inclusive a dos tercios de todas las reservas brasileñas. Hecho del cual tuvo noticia el gobierno, que fuera notificado como importante hallazgo, lo mismo que la Agencia Nacional del Petróleo, Gas Natural y Biocombustíveis (ANP), como es obligatorio por ley. Si bien se trata de una reserva gigante, los geólogos de Petrobras no tienen aún la dimensión exacta de las reservas de petróleo de Pré-Sal. Tampoco se conoce la extensión de la formación de Pré-Sal y del petróleo subyacente en la misma, la cual se estima tiene más de 100 millones de años. Puede ser que el área con potencial se extienda después del estado de Espírito Santo, que llegue al estado de Sergipe, por ejemplo. Algunos dicen que la sal gema de aquella región es de la misma naturaleza que las capas que cierran los reservatorios de petróleo descubiertos bajo el mar en Campos y Santos.

\section{EL MODELO ENERGÉTICO: REFORMAS LIBERALES Y LAS PROPUESTAS DE LULA EN 2002}

Antes de la elección de 2002 hubo un enorme debate en el país sobre las formas de apropiación de la energía por la sociedad. Básicamente había dos puntos de vista antagónicos:

El primero, buscaba encuadrar esa apropiación del petróleo dentro de un plan de ideas liberales más amplio, que implicaba una reestructuración de la producción en la economía del capital financiero, en el fondo era la respuesta a la crisis del modelo de desarrollo capitalista de tipo keynesiano, con gran intervención estatal y que, después de los logros iniciales en los años del llamado milagro de la posguerra, se estancaría. En Brasil, esa reorganización comenzó tardíamente con el gobierno de Collor de Melo y se desarrolló más ampliamente con Fernando Henrique Cardoso (FHC). En los servicios públicos, la reorganiza- 
ción consistió en la privatización de varios sectores como el de las telecomunicaciones, parte del sector eléctrico, y en el caso del petróleo, con la venta de gran parte de las acciones de Petrobras, con la tentativa de cambiar su nombre a Petrobrax, con el fin de facilitar su internacionalización. También se crearon las agencias de regulación de esos servicios, Agencia Nacional del Petróleo; Gas Natural y Biocombustibles-ANp; la Agencia Nacional de Energía Eléctrica-ANEel; la Agencia Nacional de Telecomunicaciones-ANATEL, para garantizar la rentabilidad de las inversiones, para atraer el capital internacional.

El segundo, consistiría en el control social de los servicios públicos como soporte del modo de vida urbano que emergía de la Segunda Revolución Industrial, al lograr de este modo un saneamiento de las telecomunicaciones, del servicio de energía eléctrica y de los transportes. La pregunta que surgía era ide qué forma hacer ese control? Se puede decir que en ese campo había dos corrientes. Una, la de volver al esquema de subsidios y tarifas sociales para mantener esos servicios a precios más bajos, y reducir el costo de reproducción de la fuerza de trabajo. La otra, de cierto modo reconocía ciertas reglas de mercado, que ya estaban operando pero buscaba la apropiación de parte de la renta proveniente del uso de los recursos naturales que permitía mucha más productividad del trabajo humano incorporado, en especial de los potenciales hidráulicos y los yacimientos de petróleo, así como controlar en beneficio del interés social los monopolios en la prestación de servicios.

Esta visión de usar mecanismos de mercado y apropiarse de ciertas rentas de servicios públicos fue la que al final prevaleció. Se decidió aprovechar las llamadas rentas hidráulica y petrolera, por ejemplo, para crear las bases de un cambio en el modelo liberal. Había fundamentos para eso. A pesar de las privatizaciones, gran parte del sector eléctrico - más de $80 \%$ de la generación, por ejemplo, estaba todavía en manos del gobierno. Lo que se proponía era que las empresas vendieran la energía a un precio apenas un poco abajo del valor del mercado, y la diferencia entre ese precio y el costo más bajo de la generación se destinara a un fondo social.

En el área de petróleo, donde se tenía todavía el control de Petrobras, se propuso cambiar el sistema de concesión de petróleo, en el gobierno de Fernando Henrique Cardoso (FHC). El libro sobre la Reconstrucción del sector eléc- 
trico brasilero ${ }^{7}$ sugirió introducir el sistema de producción compartida, sin embargo, esto no sucedió. Faltó voluntad política para enfrentar los intereses de las petroleras internacionales y las privadas brasileñas, así como del capital financiero para cambiar el régimen. En 2002 se creía que todavía había un riesgo significativo en la exploración, por lo que la forma de los contratos de producción compartida todavía era válida y superior a los contratos de concesión vigentes. El gobierno pasó cinco años ignorando las protestas que exigían cambiar el modelo construido por los liberales.

En el sector eléctrico, un proceso de discriminación de las empresas estatales llevó al mercado de energía, con precios extremamente favorecidos para los grandes consumidores, a un crecimiento extraordinario de cerca de $25 \%$ del consumo total de energía del país, lo que permitió la apropiación del excedente por un grupo restringido de empresas. En el caso del petróleo, en éste se mantuvo intacto el sistema de concesiones, a pesar de las amplias propuestas presentadas por políticos, juristas, profesionales de Petrobras y sindicalistas, quienes protestaron en todas las rondas de licitación de los bloques de petróleo promovidas por la ANp bajo el gobierno de Lula.

Así, la discriminación contra Petrobras era clara en la licitación, pues había una cláusula discriminatoria contra la empresa, porque en ésta se limitaba la proporción de bloques que ella podía adquirir. Se sabía también que grandes sectores del gobierno luchaban para mantener las reglas neoliberales, a pesar de varios descubrimientos ya realizados en el área del Pré-Sal. A pesar del aumento de las presiones para cambiar el régimen de exploración de petróleo se desarrolló el proyecto, en noviembre de 2007, la Novena Ronda de Licitación, los bloques fueron escogidos, anunciados y la licitación mantenida hasta la víspera de su realización. Así, finalmente, el presidente reunió al Consejo Nacional de Políica Energética (CNPE) y se retiraron de la licitación los 41 bloques en torno del campo petrolero de Tupi. Sin embargo, se mantuvieron 11 bloques del arco de Cabo Frío, rematados por la oGX, que, sin reacción del gobierno, meses antes habían reclutado técnicos de Petrobras, poseedores de informaciones estratégicas y privilegiadas.

7 Sauer et al., A reconstrução do setor eletrico brasileiro, São Paulo, Paz e Terra, 2003, 300 pp. 


\section{LA PROPUESTA DEL GOBIERNO: ANÁLISIS CRÍTICO}

Después del descubrimiento anunciado en 2007, a mediados de 2008 el gobierno nombró una Comisión Interministerial (Ministerio de Minas y Energía; Ministerio de Desarrollo Industrial y Comercio Exterior; Casa Civil; Ministerio de Hacienda; y Ministerio de Planeación, Presupuesto y Gestión) para elaborar nuevas leyes para el sector, relativas a la producción de petróleo y gas en el área de Pré-Sal. ${ }^{8}$ En agosto de 2009 la Comisión dio a conocer cuatro proyectos de ley para reformar la legislación del sector petróleo: el Proyecto de Ley de la Cámara PLC-309/09, que crearía la Empresa Brasileña de Administración de Petróleo y Gas Natural S. A. -Petro-Sal-; el PLC 7/10, que crea el Fundo Social (FS); el PLC 8/10, que establece reglas para la capitalización de Petrobras; y el PLc 16/10, que establece nuevos criterios para la distribución de los royalties del petróleo y crea el régimen de producción compartida. ${ }^{?}$

${ }^{8}$ Brasil, 2008. Decreto de 17 de julho de 2008. Institui Comissão Interministerial com a finalidade de estudar e propor alterações necessárias na legislação, no que se refere à exploração e à produção de petróleo e gás natural nas novas províncias petrolíferas descobertas em área denominada Pré-Sal. Diário Oficial da União-DOU, Brasilia, 18 de julio, 2008, sec. 1.

9 Brasil, 2009. PLC-ProjeTo DE LEI DA CÂMARA, num. 309, 2009. Autoriza o Poder Executivo a criar a empresa pública denominada Empresa Brasileira de Administração de Petróleo e Gás Natural S. A. - PETRO-SAL e dá outras providências. Brasilia, 27 de noviembre, 2009.

Brasil, 2010. PLC-PROJETO DE LEI DA CÂMARA, num. 7, 2010. Cria o Fundo Social - Fs; dispõe sobre sua estrutura e fontes de recursos; altera a Lei num. 9.478, 6 de agosto, 1997; e dá outras providências. Brasilia, 8 de marzo, 2010.

Brasil, 2010a. PLC-PROJETO DE LEI DA CÂMARA, num. 8, 2010. Autoriza a União a ceder onerosamente à Petróleo Brasileiro S. A. - PETROBRAS o exercício das atividades de pesquisa e lavra de petróleo, de gás natural e de outros hidrocarbonetos fluidos de que trata o inciso I do art. 177 da Constituição Federal, e dá outras providências. Brasilia, 11 de marzo, 2010.

Brasil, 2010b. PLC-PROJETO DE LEI DA CÂMARA, num. 16 de 2010. Dispõe sobre a exploração e a produção de petróleo, de gás natural e de outros hidrocarbonetos fluidos sob o regime de partilha de produção, em áreas do Pré-Sal e em áreas estratégicas, altera dispositivos da Lei num. 9 478, de 6 de agosto de 1997, e dá outras providências. (Exploração e produção de petróleo; competências do CNPE, da ANP e do Ministério de Minas e Energia; casos de contratação direta e de licitação para exploração de petróleo; contratos de partilha de produção; rateio das rendas governamentais no regime de partilha de produção (royalties); comercialização do petróleo.). Brasilia, 22 de marzo, 2010. 
En los cuatro proyectos de ley, el gobierno prácticamente acogió todas las sugerencias de los movimientos sociales, pero dejó todo abierto para luego no ejecutar ninguna de ellas. Los contratos de producción compartida eran una propuesta y una solución en 2002, cuando Lula fue elegido. Hoy, la situación es en apariencia otra. Las nuevas leyes recolocan a Petrobras al mando del proceso de exploración, producción y venta de petróleo en el país.

Actualmente estos proyectos de ley son tramitados en el Congreso en el régimen de urgencia y con prioridad de votación. El 10 de junio de 2010 fueron aprobados el PLC $7 / 10$ que crea el Fondo Social y el PLC 8/10, que autoriza la capitalización de Petrobras. A pesar de recibir mayoría favorable, el PLC 7/10 retornará para análisis de la Cámara dado que el texto aprobado incluyó, por petición de uno de los senadores, la propuesta del plc 16/10 sobre la adopción del régimen de producción compartida para la exploración del petróleo de Pré-Sal. La explicación de juntar el PLC 7/10 y el PLC 16/10 fue considerar que el Fondo Social es parte integrante del régimen de producción compartida, ya que la mayor parte de sus recursos sería proveniente de los ingresos recibidos por la comercialización del petróleo, propiedad del Gobierno Federal. Otra de las medidas votadas y aprobadas fue la enmienda que distribuye los royalties del petróleo entre todos los estados y municipios, y que a su vez establece que el Gobierno Federal compensará a los estados productores - Río de Janeiro y Espírito Santopor la pérdida de recursos. Algunos representantes del gobierno esperan que el presidente de la República vete esta enmienda. ${ }^{10}$

La razón de por qué la votación de los proyectos sucede de forma acelerada, parecería radicar en que el gobierno necesita tener los recursos petroleros para dedicarlos, lo más rápido posible, al Fondo Social que una de las cuatro leyes explicadas establece. Pero esto es cuestionable. Suponiendo que todo suceda de forma acelerada: 1) que la legislación nueva sea aprobada; 2) el gobierno promueva las licitaciones y Petrobras y otras empresas, con el nuevo régimen legal, o de producción compartida del petróleo obtenido, disputen los bloques de

\footnotetext{
${ }^{10}$ Agencia Senado, 10 de junho de 2010. Senado aprova criação do Fundo Social do Pré-sal, regime de partilha e distribuição dos 'royalties' a todos os estados. En http://www.senado.gov.br/agencia/verNoticia.aspx?codNoticia $=102781 \&$ codAplicativo $=2$ (fecha de consulta: junio de 2010).
} 
Pré-Sal; 3) en 3 o 4 años más, después de la firma de los contratos, las empresas exploren sus áreas y hagan las instalaciones para colocar los pozos en producción; 4) en los 3 o 4 años siguientes, ellas se dediquen, según lo establecido en el proyecto de ley, a producir el petróleo necesario para el llamado, "óleo costo" que ellas pagarán solas; 5) entonces, finalmente, cuando la producción comience a ser repartida, la parte del gobierno irá, en primer lugar, para el Fondo de Desarrollo Social, y, después, cuando éste fondo dé frutos, sus rendimientos serán distribuidos en las actividades económicas y sociales determinadas. Si todo lo anterior sucede conforme a lo planeado, cerca del año 2018 o 2020, los recursos aparecerán en el Fondo. Tal vez en 2022, sus rendimientos tendrán una escala importante para financiar la "nueva independencia" del país. Por eso la prisa levanta dudas. Otro factor puede ser las elecciones que están muy próximas y esas nuevas licitaciones significan grandes negocios. El afán de las grandes petroleras internacionales en tomar parte del derecho de las grandes reservas de petróleo es visible. ${ }^{11}$ Es fácil comprenderlo: sus reservas son una fracción mínima comparada con las de 1960, cuando, de cierto modo, mandaban en el mundo. Sin embargo, esa prisa no es interesante para el país. Por ello, se debe hacer una evaluación más precisa del petróleo que todavía no ha sido licitado. Es esencial la contratación de Petrobras para concluir el proceso exploratorio, es decir, conocer las acumulaciones, sus límites, desarrollar un plano de evaluación y, posteriormente, la producción. Así, se sabrá con certeza si hay 80 000, 100 000, 200000 o más millones de barriles.

Sólo así se podrá planear la producción. No se puede olvidar que la propia OPEP no produce sin calcular. Ella articula el equilibrio de oferta y demanda y tiene como precio-objetivo estratégico el petróleo entre 60 y 80 dólares el barril. Las formas básicas de operar la industria del petróleo -monopolio público ope-

${ }^{11}$ Según Valor Económico, días después de la divulgación de la respuesta de la nueva legislación, el presidente de la República estuvo en Nueva York, donde grandes empresarios le ofrecieron una cena para tratar sobre inversiones en el país. El acontecimiento costó más de 700 mil dólares. Dos empresas del sector petrolero, una brasileña, la OGX, y otra multinacional, la Exxon Mobil, pagaron la mayor parte de la cuenta: cada una de ellas 200000 dólares. R. Balthazar, "Exxon Mobil busca proximidade com Lula para influenciar no Pré-Sal", en Valor Económico, Nueva York, 23 de septiembre, 2009. 
rado por empresa estatal o la contratación para la prestación de servicios, ${ }^{12}$ producción compartida ${ }^{13}$ y concesión de áreas $-{ }^{14}$ ya fueron ampliamente explicadas. Sin embargo, no se destacó lo siguiente: el monopolio público ejercido por la operadora estatal es la forma más simple y más utilizada. Es el régimen adoptado por Arabia Saudita y por todos los otros países con grandes reservas, como Irán y Venezuela. Cuando es necesario subcontratan la prestación de servicios, y raramente la producción compartida. Los regímenes de producción compartida y el de concesiones fueron dominantes en otra época, en la década de 1960,

${ }^{12}$ Este modelo envuelve la contratación de una empresa para la realización de la actividad de exploración, con la entrega de la producción al gobierno. Generalmente las empresas actúan con contratos de riesgo y son remuneradas cuando los campos entran en producción. Los servicios de la compañía pueden ser pagados en petróleo crudo o en dinero. Instituto Brasileiro do Petróleo (IBP), 2010. Informativo sobre o Pré-Sal: modelos de contrato. En http://www.ibp.org. $\mathrm{br} /$ main.asp? Team $=\% 7 \mathrm{BF} 400 \mathrm{BB} 19-\mathrm{AB} 8 \mathrm{~B}-4 \mathrm{DD} 4-97 \mathrm{~F} 0-006 \mathrm{C} 250 \mathrm{~A} 46 \mathrm{C} 3 \%$ 7D (fecha de consulta: mayo de 2010).

${ }^{13}$ En 1966 Indonesia introdujo los contratos de producción compartida (Barrows, 1980, apud Adriana Elvira Barrios, "Evaluación de los regímenes fiscales de petróleo en Colombia", en Astrid Martínez, ¿Es atractiva la contratación petrolera para la inversión privada en Colombia?, Bogotá, unat, 2005, pp. 15-62). Este tipo de contrato tiene tres elementos: uno, la recuperación de costos, es decir, un porcentaje de los ingresos de la venta de petróleo se aparta para pagar los costos de exploración y producción de la empresa. Dos, después de deducir la reducción de costos se divide la producción en proporciones predeterminadas entre el contratista y el gobierno, usualmente por medio de su empresa petrolera nacional. Tercero, el impuesto a la renta, pues la mayoría de las empresas están sujetas a pagar los impuestos de cada país sobre su participación en la producción (Barrios, op. cit.). En el régimen de producción compartida, el petróleo y el gas natural producidos son divididos entre el Gobierno Federal y las empresas que exploran los campos. Por la propuesta que altera la Ley núm. 9.478/97 (PLC, $\left.\mathrm{N}^{\circ} 16 / 10\right)$, sobre la política energética nacional, se establece el marco regulatorio del sector, el gobierno podrá contratar a Petrobras o a otras empresas, a través de licitación, para retirar el petróleo de los yacimientos. Vence quien ofrezca el mayor porcentaje de petróleo extraído para el Gobierno Federal. Petrobras es asegurada con un porcentaje mínimo de $30 \%$ de cada bloque licitado.

${ }^{14}$ La empresa adquiere el derecho de explorar un área mediante el pago, como contrapartida de regalías e intereses al gobierno. En Brasil, la transferencia de las concesiones a las empresas es hecha por la ANP a través de licitaciones de bloques exploratorios. A cambio del derecho de explorar las áreas adjudicadas por licitación, las empresas remuneran al Estado por medio de bonos, regalías, o la participación especial. Instituto Brasileiro do Petróleo (IBP), 2010. Informativo sobre o Pré-Sal: modelos de contrato. En http://www.ibp.org.br/main. asp?Team=\%7BF400BB19-AB8B4DD4-97F0-006C250A46C3\%7D (fecha de consulta: mayo de 2010). 
cuando las grandes multinacionales del petróleo, las llamadas Siete Hermanas -Shell, Esso, British Petroleum y otras - tenían cerca de 90\% de las reservas mundiales, en comparación con 10\% que tienen hoy. ${ }^{15}$

Actualmente, las Siete Hermanas del petróleo, como citó el Financial Times, son las compañías nacionales: 1) Saudi ARAMCO; 2) Gazprom, rusa; 3) CNPC, china; 4) NIOC, iraniana; 5) PDVSA, venezolana; 6) Petrobras, brasileña, y 7) Petronas, Malasia. ${ }^{16}$ El régimen del monopolio público ejercido por operadora estatal pasó a ser adoptado en la medida en que los países más pobres se fueron dando cuenta del enorme excedente generado por el petróleo y de la necesidad de controlarlo.

No se puede definir un plan de producción para el petróleo de Pré-Sal sin conocer esa reserva de forma precisa. Por ello, la primera decisión que debería tomarse sobre los campos gigantes de petróleo de Pré-Sal es la contratación de Petrobras, la que los descubrió, para evaluar toda su extensión, mediante un contrato con el gobierno por el costo del servicio. El petróleo es, cada vez más, un recurso geopolítico. Las grandes reservas mundiales están bajo el control de los estados nacionales y de sus empresas estatales. Actualmente la producción mundial de petróleo es de 80 millones de barriles por día. ${ }^{17}$ Brasil tiene 100000 millones de barriles en la capa Pré-Sal, que es más o menos lo que se está evaluando, en opinión de varios analistas. Si se decide explotar esa reserva en 30 años, Brasil pondrá en el mercado alrededor de 10 millones de barriles por día, más o menos como Arabia Saudita en la actualidad. Pero Arabia Saudita no deja decidir al mercado por ella. Ayudó a formar la OPEP. ¿Por qué? Porque la entrada de un actor importante en el mercado mundial del petróleo tiene consecuencias sobre los precios. ¿Cuál es el precio del petróleo hoy en día?

Antes de esta crisis, se decía que los biocombustibles tendrían un espacio, pero que la amenaza de una crisis internacional bajaría los precios del petróleo, sin embargo, llegó la crisis y el precio del petróleo está alrededor del intervalo-objetivo

${ }^{15}$ Business Week (BW), The Changing Game, 15 de mayo, 2006. En http://www.businessweek.com/ magazine/content/06_20/b3984005.htm (fecha de consulta: septiembre de 2009).

${ }^{16} \mathrm{C}$. Hoyos, "The new Seven Sisters: oil and gas giants dwarf western rivals", en Financial Times, Londres, 11 de marzo, 2007.

${ }^{17}$ British Petroleum, op. cit. 
de la OPEP, entre 60 y 80 dólares por barril. Esto confirma la tesis de que el petróleo sigue siendo de gran valor, es robusta. También refuerza la hipótesis de que la extracción del petróleo del subsuelo y su conversión en moneda, cualquiera que ella sea - dólares o yuanes- puede no ser inteligente. Hoy, por ejemplo, si el dinero obtenido de la explotación del petróleo fuera destinado a ser parte de las reservas monetarias brasileñas, éste sería un mal negocio. El dólar se compra con títulos de la deuda pública para no causar inflación interna, con la tasa de interés de Brasil - la tasa Selic a $8.5 \%$ por año-y fuera se compran títulos del tesoro de Estados Unidos que están pagando menos de $4 \%$ al año. Los dólares también podrían tener otras aplicaciones.

El fondo soberano que se pretende constituir, con uno de los proyectos de ley enviado por el gobierno al Congreso, podría, por ejemplo, comprar una gran parte de las acciones de Petrobras que ahora están bajo control extranjero. Pero tampoco esto es un buen negocio ahora, que está cambiando el marco regulatorio del petróleo en el país: después del cambio, probablemente, el precio de la acción valga menos. La valorización de las acciones de Petrobras depende de la evolución de su capacidad de producción, de su tasa de nuevos descubrimientos y de su capacidad de convertir estos factores en los beneficios futuros. Pero depende crucialmente de cuan obediente es Petrobras a las reglas del mercado financiero, y en qué medida el gobierno brasileño se mantenga fiel a la ortodoxia financiera. La pregunta que surge es si chabrá la capacidad de entender estas cosas, de construir un camino propio o si se continuará obedeciendo la normatividad de las grandes empresas? No cabe duda de que el capital financiero está ahí, en la búsqueda de negocios rentables. Quieren que el gobierno se comporte dentro de estas reglas.

Para escapar de este destino, el país debe tener un proyecto nacional de desarrollo social y económico, un plan que incluya la educación. Pero si no hay planes, no existe tampoco la capacidad de gestión para aplicarlos en la dimensión necesaria, que sería de 20 a 30000 millones de dólares por año. El plan también debe incluir la salud, que está vinculada a la prevención, saneamiento, infraestructura y medio ambiente urbano. En esta área existe el Sistema Único de Salud (sus) que, como concepción, es un proyecto de referencia que apunta a dónde 
ir pero no hay recursos para llegar allí. El modelo agrícola brasileño también tendría que ser reconsiderado. Se necesita incorporar a la gran masa de población que continúa viviendo en zonas rurales y mejorar la planificación de la ocupación del territorio brasileño, incluso en grandes extensiones sin explorar o mal exploradas, depredadas, como el Amazonas. El plan debe abordar la infraestructura, sobre todo la de circulación, dentro del área urbana y entre las áreas económicas y geográficas del país. El sistema actual es lo peor posible: muy intensivo en uso de la energía, contaminante e ineficaz. Necesita ser cambiado para valorizar el transporte ferroviario, fluvial, la navegación costera y el metro urbano. Como se puede ver, un plan nacional de desarrollo económico y social requiere un gran esfuerzo. Y el país no está preparado para ello. Prueba de esto es que el Ministerio de Planeación actual es simplemente un órgano de ejecución y supervisión del presupuesto. El gobierno no tiene un plan estratégico.

Lo que el gobierno presentó para tratar el petróleo de Pré-Sal en los cuatro proyectos de ley enviados al Congreso, básicamente, concede al Ejecutivo, en especial al presidente de la República, y a algunos segmentos del Ejecutivo, dependientes de la presidencia de la República, el poder de arbitrar el acceso a la exploración de Pré-Sal. Presentó cuatro proyectos de ley y no sólo uno para dividir el foco del problema y resaltar el papel de coordinador central, que es el Ejecutivo.

El primer proyecto es el que establece el régimen de producción compartida. Éste fue relevante en 2002, no lo es más. Ahora, la producción compartida sirve para mantener la sensación de que existe riesgo en el proceso de exploración de Pré-Sal. Si el Estado brasileño no sabe administrar ese riesgo, debe por lo tanto llamar a los especialistas en la administración de riesgo, es decir, al gran capital extranjero. Pero ello está lejos de la verdad a la luz de los reales peligros de exploración del petróleo. Podemos dividir éstos en cuatro categorías: a) el geoló gico-geofísico, para la exploración de las áreas para localizar el petróleo; b) el tecnológico y de ingeniería, para determinar las estructuras de producción que serán instaladas en las áreas para extraer petróleo; c) el financiero, para reunir capitales y promover la inversión, y d) el riesgo comercial, que tiene que ver con la comercialización del petróleo en un mercado complejo. El riesgo exploratorio en el caso del petróleo de Pré-Sal es pequeño y puede ser prácticamente 
eliminado, si hay interés. Es más, en el proyecto de Ley de la Producción Compartida, el gobierno admite que puede reducir ese riesgo. La ANp se encargó de perforar algunos pozos en Pré-Sal, con el fin de evaluar las reservas y para eso la agencia ya está tratando con Petrobras. Además, en la ley enviada al Congreso, en su artículo 7, el gobierno estableció que el Ministerio de Minas y Energía podrá hacer la evaluación previa de los yacimientos. Es claro que no debería ser "podrá"; debería ser: el ministerio "deberá" hacer la evaluación previa de los yacimientos. La evaluación lleva, en promedio, de dos a tres años. De esta forma se puede delimitar mejor el Pré-Sal y saber, por ejemplo, si se extiende hasta el estado de Bahia, o hasta Sergipe.

Desafortunadamente, el "podrá" que está en el proyecto de Ley de Producción Compartida está alineado con todo el resto del marco regulatorio anunciado. Ésta es una especie de estructura circular. Parece que todo está institucionalizado, se escucha el Consejo Nacional de Política Energética, la nueva empresa: la Petro-Sal, la ANP. Pero, en el fondo, todo está centralizado en el poder del presidente, todos los demás organismos pueden ser dispensados en cualquier momento por el presidente de la República. Pocas veces en la historia del país intervino el Congreso para refrendar un apoderamiento de tan elevado impacto económico. Si fueran los 100000 millones de barriles que muchos suponen, éstos serán explotados en 30 años, con ingresos cercanos a 1000 millones de dólares por día.

El papel atribuido a Petro-Sal refuerza el carácter centralizador y arbitrario del conjunto de proyectos. ¿Cómo funciona la industria del petróleo, en el caso de las licitaciones? Un consorcio de empresas o una empresa única concurre en ellas. Si es sólo una, o ella es la propia operadora, o contrata una operadora escogida por ella. Si es un consorcio, los socios se ponen de acuerdo y escogen una operadora. Hacen un Joint Operation Agreement (JOA), que es el instrumento por el cual se definen las reglas de operación. El operador toma las decisiones de explotación, cuántos pozos van a perforarse, en qué plazos, y somete algunas decisiones a los miembros del consorcio. Luego realiza el "cash call", que es llamar al capital para las inversiones. De acuerdo con las reglas de la legislación enviada al Congreso, si Petrobras gana la licitación, ella decidirá lo que va a realizar junto con Petro-Sal. 
Para ello existirá un comité operativo para cada área licitada, donde Petro-Sal tiene la mitad de los votos además del poder de veto. Petro-Sal podrá, en última instancia, decidir mejor que Petrobras en todas las cuestiones asociadas al proyecto de aprovechamiento de los recursos del bloque licitado. Son decisiones que involucran un conocimiento sobre una enorme gama de temas como geología, ingeniería, financiamiento, comercio mundial de petróleo, entre otros.

El gobierno cree que Petro-Sal, una empresa que nacerá ahora con un conjunto de profesionales ya definido como pequeño, debe tener la última palabra y no Petrobras, debido a que existe en su núcleo central desconfianza hacia éste. La situación resulta todavía más compleja si, en las licitaciones, Petrobras hace una propuesta que pierde por lo que, como manda la ley, tiene que quedar obligatoriamente como operadora y con 30\% del capital de inversión en el bloque licitado.

Toda esa confusión resulta del hecho de que el gobierno, en cierto modo, haya aceptado todas las críticas y, en principio, haya restablecido a Petrobras en el estratégico papel de exploración de petróleo en el país. Pero, en la práctica, haberlo hecho de modo ambiguo, ha permitido hacer exactamente lo contrario. Ese bloque puede no ser licitado y puede ser entregado directamente a Petrobras, la cual va a ser la operadora de todos los campos y la idea es que va a contratar a los proveedores y a ampliar el pedazo nacional de los negocios. Petrobras va a ser, posiblemente, la empresa escogida para comercializar el petróleo. Pero podría no serlo. Petro-Sal podría decidir que la Exxon, la Mobil, o la ogx, son las que comercializarán el petróleo.

Esa incertidumbre tiene claramente un propósito. Tener la idea de que existe riesgo, abre el espacio para los grandes operadores de riesgo que son los del sistema financiero. El país echa mano de su proyecto, de su plan y se mantiene bajo el control del sistema internacional. La tabla 1 muestra en detalle los números del balance de Petrobras. Lo cual permite tener una radiografía de lo sucedido actualmente en el sector petrolero en Brasil, cuando la estatal tiene el cuasi monopolio de la producción. Ayuda a ver cómo podría quedar en el futuro, mediante el sistema híbrido que el gobierno pretende establecer. Como se sabe, el gobierno quiere mantener la apertura establecida para el capital privado con 
Ildo Sauer, Sonia Seger y Julieta Puerto Rico

el sistema de concesiones creado en 1997. Para las áreas de Pré-Sal y otras consideradas estratégicas, se pretende establecer el sistema de producción compartida de la producción.

Tabla 1

LAS CUENTAS DE PETROBRAS DE 2008

(1 000 millones de reales)

\begin{tabular}{|l|r|}
\hline INGRESOS & 316 \\
\hline GASTOS & 174 \\
\hline * Materiales consumidos & 48 \\
\hline * Energía y servicios & 52 \\
\hline * Materiales comprados para reventa & 53 \\
\hline * Depreciaciones y amortizaciones & 11 \\
\hline * Otros gastos & 10 \\
\hline VALOR ADICIONADO & 142 \\
\hline * Personal (salarios/benef./FGTS) & 15 \\
\hline * Gobierno (impuest./tasas/contrib.) & 85 \\
\hline * Lucros y dividendos, de los cuales 12 billones \\
son del Gobierno Federal
\end{tabular}

Fuente: Petrobras, 2009.

Del balance, se destaca, en primer lugar, el valor líquido adicionado por Petrobras, 97000 millones de reales que se pagan como renta a los gobiernos. Ese número corresponde a 85000 millones - transferidos al Gobierno Federal por cuenta de regalías, participaciones especiales y bonos exigidos en los contratos de concesión actuales-, 12000 millones más, de lucros y dividendos repasados al Gobierno Federal por cuenta de 40\% de acciones que tiene de la empresa. La suma corresponde a casi $70 \%$ de toda la renta extra agregada por la Petrobras. 
Así, para cada uno de los 800 millones de barriles producidos en $2008,{ }^{18}$ cerca de $R \$ 125$ o US $\$ 70$ por barril fueron apropiados públicamente. Esto significa que el país, así mantenga la legislación actual, debe aumentar, en mucho, los ingresos del gobierno, gracias al crecimiento de la producción con los yacimientos de Pré-Sal. En una escala de países con las mayores reservas de petróleo, Arabia Saudita está en primer lugar, con 264000 millones de barriles. Brasil estaba en el puesto 17, con 14000 millones de barriles. Con los primeros descubrimientos de Pré-Sal, Brasil pasó a 20000 millones, y quedó en el lugar 12, después de Estados Unidos. ${ }^{19}$ La pregunta es icuánto petróleo existe en Pré-Sal?, ¿80 000 millones de barriles?, ¿100 000?, ¿200 000 millones? Incluso sin Pré-Sal se producen 2 millones de barriles de petróleo por día. De existir 100000 millones de barriles se podría producir petróleo por 30 años (10 millones de barriles por día), cuando en el mundo la era del petróleo ya esté declinando para entonces. A partir de las cuentas de Petrobras ya citadas, el potencial con que se cuenta significaría un ingreso estatal extra cinco veces mayor que el de 2008 que fue de 97000 millones. Es decir, cerca de 500000 millones de reales por año, un número próximo de todo lo recaudado actualmente de impuestos en Brasil, que es de $800000 \mathrm{mi}-$ llones de reales. Hay consenso, incluso con las grandes petroleras extranjeras, de que la fracción que cabe a los gobiernos puede aumentar en los nuevos contratos. Si esa participación estatal aumenta del actual 70\% ya citado, a 80\% o hasta $90 \%$, por un aumento de las participaciones especiales en los contratos de concesión, como prefieren las petroleras, o por el sistema de producción compartida propuesto por el gobierno.

Sin embargo, esto no resuelve el problema de la apropiación de renta del petróleo. Hoy, los gobiernos destinan esos recursos para cumplir metas del superávit primario, pagar los intereses de su enorme deuda interna y para otros fines, que no constituyen, mínimamente, un plan estratégico para modificar el patrón de desarrollo del país. La multiplicación de esos recursos, por el aumento

\footnotetext{
${ }^{18}$ Balance Energético Nacional 2009, datos de 2008.

${ }^{19} \mathrm{~J}$. Chang, Brazil's Big Find. Massive deep-water oil find in Brazil challenges technology, Río de Janeiro, McClatchy Newspapers, 2007. En http://www.mcclatchydc.com/2007/12/01/22225/ massive-deep-water-oil-find-in.html\#ixzz0nYUME2cY. (fecha de consulta: septiembre de 2009).
} 
de la cantidad de petróleo a producirse con los yacimientos de Pré-Sal puede, incluso, agravar algunos problemas de la economía brasileña. En primer lugar, por la clara perspectiva de una mayor participación en el sector por parte de empresas extranjeras y privadas, supuestamente nacionales, pero asociadas de modo estrecho con el capital extranjero.

\section{CONCLUSIONES}

La propuesta básica es eliminar la especulación sobre las reservas de Pré-Sal y cuantificarlas para después explotarlas de acuerdo a un plan nacional de desarrollo, previamente diseñado.

El modelo de producción compartida, propuesto por el gobierno, no cuenta con el apoyo de los movimientos sociales, defensores de la campaña "El petróleo tiene que ser nuestro", sometido al Congreso, con suscripción de más o menos dos decenas de diputados, un proyecto alternativo.

La evaluación de los movimientos sociales es que no faltarán recursos ni financieros ni tecnológicos, pues el control de las reservas de petróleo garantiza el financiamiento necesario para su producción por Petrobras, detentora de la mayor capacitación en el área de Pré-sal y con acceso garantizado a todas las tecnologías de punta disponibles en el mundo.

El modelo propuesto por los movimientos sociales es: a) la conclusión del proceso exploratorio, mediante contrato con Petrobras, para la medición y evaluación de las reservas; b) la restauración del monopolio estatal del petróleo; c) la reestatización de 100\% de Petrobras mediante recompra de acciones y capitalización con reservas; d) el desarrollo de un plan nacional de desarrollo económico y social que incluya: educación, salud, urbanización, habitación, saneamiento, movilidad, inclusión digital, puertos, vías acuáticas, ferrovías, trenes urbanos, ciencia y tecnología, desarrollo y reforma agraria, y recursos para promover la transición energética sostenible, y e) planear la producción de petróleo en el ritmo necesario a la capitalización del Fondo Social para financiar tal plan.

Finalmente, para tratar debidamente la nueva situación creada por el descubrimiento de petróleo de Pré-sal, que puede tener repercusiones extraordi- 
narias en la vida de los brasileños, se propone un plebiscito, a través del cual, las fuerzas progresistas del país creen profundizar la comprensión del punto central: que las extraordinarias rentas que el petróleo propicia hoy $-\mathrm{y}$ debe continuar propiciando en las próximas dos o tres décadas, por lo menos- debería servir a un nuevo plan de desarrollo económico y social, capaz de modificar a Brasil de manera radical. Esto sería concomitante con las elecciones de 2010, con dos preguntas:

1) ¿Debe el Gobierno Federal retomar y ejercer el monopolio sobre el petróleo y promover tanto su extracción como su producción, vinculadas exclusivamente al financiamiento de un plan nacional de desarrollo económico y social?

2) ¿Debe Petrobras ser reestatizada y ejecutora del monopolio?

Recibido: 20 de mayo, 2010. Aceptado: 15 de junio, 2010.

BibLIOGRAF́́A

AgenCia Senado. 10 de junho de 2010. Senado aprova criação do Fundo Social do Pré-sal, regime de partilha e distribuição dos 'royalties' a todos os estados. En: http://www.senado.gov.br/agencia/verNoticia.aspx?codNoticia $=102781 \&$ codAplicativo = 2 (fecha de consulta: junio, 2010).

Balthazar, R., "Exxon Mobil busca proximidade com Lula para influenciar no Pré-Sal", en Valor Econômico, Nueva York, 23 de septiembre, 2009.

BarRios, Adriana Eivira, "Evaluación de los regímenes fiscales de petróleo en Colombia", en Astrid Martínez, iEs atractiva la contratación petrolera para la inversión privada en Colombia?, Bogotá, unAL, 2005, pp. 15-62. 
BRASIL, 2008. Decreto de 17 de julho de 2008. Institui Comissão Interministerial com a finalidade de estudar e propor alterações necessárias na legislação, no que se refere à exploração e à produção de petróleo e gás natural nas novas províncias petrolíferas descobertas em área denominada Pré-Sal. Diário Oficial da União-DOU, Brasilia, 18 de julio, 2008. Seç. 1.

BRAsil, 2009. PlC-Projeto de leI dA CÂmarA, num. 309, 2009. Autoriza o Poder Executivo a criar a empresa pública denominada Empresa Brasileira de Administração de Petróleo e Gás Natural S. A. PETro-SAL e dá outras providências. Brasilia, 27 de noviembre, 2009.

Brasil, 2010. PLC-PROJETO DE LeI dA CÂMARA, num. 7, 2010. Cria o Fundo Social - FS; dispõe sobre sua estrutura e fontes de recursos; altera a Lei num. 9.478, 6 de agosto, 1997; e dá outras providências. Brasilia, 8 de marzo, 2010.

BRASIL, 2010a. PLC-PROJETO DE LEI DA CÂMARA, num. 8, 2010. Autoriza a União a ceder onerosamente à Petróleo Brasileiro S. A. PETROBRAS o exercício das atividades de pesquisa e lavra de petróleo, de gás natural e de outros hidrocarbonetos fluidos de que trata o inciso I do art. 177 da Constituição Federal, e dá outras providências. Brasilia, 11 de marzo, 2010.

BRASIL, 2010b. PLC-PRoJeto dE IEI DA CÂMARA, num. 16 de 2010. Dispõe sobre a exploração e a produção de petróleo, de gás natural e de outros hidrocarbonetos fluidos sob o regime de partilha de produção, em áreas do Pré-Sal e em áreas estratégicas, altera dispositivos da Lei num. 9478 , de 6 de agosto de 1997, e dá outras providências. (Exploração e produção de petróleo; compe tências do CNPE, da ANP e do Ministério de Minas e Energia; casos de contratação direta e de licitação para exploração de petróleo; contratos de partilha de produção; rateio das rendas governamentais no regime de partilha de produção (royalties); comercialização do petróleo). Brasilia, 22 de marzo, 2010.

British Petroleum (BP), Statistical Review of World Energy 2010. En http:// www.bp.com/liveassets/bp_internet/globalbp/globalbp_uk_english/reports_and_publications/statistical_energy_review_2008/STAGING/ocal_a ssets/2010_downloads/Statistical_Review_of_World_Energy_2010.xils (fecha de consulta: junio de 2010). 
Business Week (Bw), The Changing Game, 15 de mayo, 2006. En http://www.businessweek.com/magazine/content/06_20/b3984005.htm (fecha de consulta: septiembre de 2009).

Chang, J., Brazil's Big Find. Massive deep-water oil find in Brazil challenges technology, Río de Janeiro, McClatchy Newspapers, 2007. En http://www. mcclatchydc.com/2007/12/01/22225/massive-deep-water-oil-findin.html\# ixzz0nYUME2cY (fecha de consulta: septiembre de 2009).

Hoyos, C., "The new Seven Sisters: oil and gas giants dwarf western rivals", en Financial Times, Londres, 11 de marzo, 2007.

Empresa de Pesquisa Energética, Brasil. Balanço Energético Nacional: ano base 2008. Río de Janeiro, EPE, 2009, 274 pp.

Instituto Brasileiro do Petróleo (iBP), 2010. Informativo sobre o Pré-Sal: modelos de contrato. En http://www.ibp.org.br/main.asp?Team=\%7BF400BB19AB8B-4DD4-97F0-006C250A46C3\%7D (fecha de consulta: mayo de 2010).

Neto, J. O. y A. J. Costa, "A Petrobras e a exploração de petróleo offshore no Brasil: um approach evolucionário", en Revista Brasileña de Economía, vol. 61, núm. 1, Río de Janeiro, enero-marzo, 2007.

Petróleo Brasileiro S. A., Petrobras 2009a. Balanço Social e Ambiental 2008, Río de Janeiro, 2009.

Petróleo Brasileiro S. A., Petrobras 2009b. Presalt Reservoir. En http://www2.petrobras.com.br/ri/pdf/Petrobras_EP_MerrillLynch.pdf (fecha de consulta: mayo de 2010).

Pinho, RicaRdo ReISEN DE, Gigantes Brasileiros: Multinacionais emergentes e Competição Global, 2008 (Tese de Doutorado. Escola de Administração de Empresas, Fundação Getulio Vargas, São Paulo).

SAuER, I.L et al., A Reconstrução do Setor Eletrico Brasileiro, São Paulo, Paz e Terra, 2003, $300 \mathrm{pp}$. 